\title{
Supporting Information - High-Throughput Automation of Endosomolytic Polymers for mRNA Delivery
}

David Ulkoski ${ }^{\dagger \dagger}$, Michael J. Munson ${ }^{\dagger} \|$ Max E. Jacobson ${ }^{\S}$, Christian R. Palmer ${ }^{\S}$, Carcia S. Carson^, Alan Sabirsh", John T. Wilson $^{\S \wedge}$, and Venkata R. Krishnamurthy ${ }^{\ddagger *}$

*Advanced Drug Delivery, Pharmaceutical Sciences, R\&D, AstraZeneca, Boston, USA. "Advanced Drug Delivery, Pharmaceutical Sciences, R\&D, AstraZeneca, Gothenburg, Sweden. $\S$ Department of Chemical and Biomolecular Engineering, Vanderbilt University, Nashville, Tennessee, USA.

${ }^{\wedge}$ Department of Biomedical Engineering, Vanderbilt University, Nashville, Tennessee, USA.

†Contributed equally to this work

*Corresponding Author:

Email: venkata.krishnamurthy@astrazeneca.com; vkrishna.medchem@gmail.com 


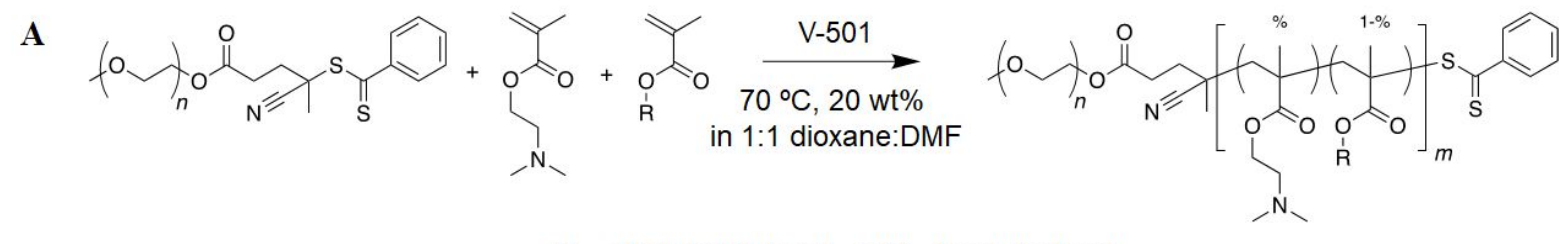

$R=$ ethyl, butyl, hexyl, octyl, decyl, dodecyl

B<smiles>[R]OC(=O)CCC(C)(C#N)SC(=S)c1ccccc1</smiles><smiles>C=C(C)C(=O)OCCCC</smiles>

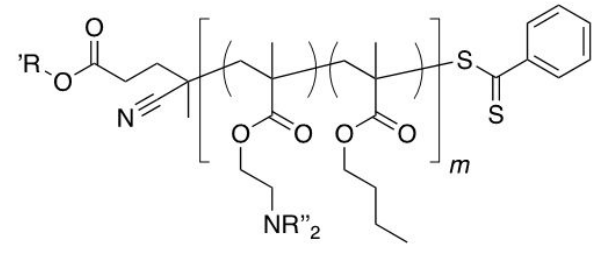

$\mathrm{R}^{\prime}=\mathrm{H}, \mathrm{mPEG}(2 \mathrm{kDa})$
$\mathrm{R}^{\prime \prime}=$ methyl, ethyl

Scheme S1. Generalized Synthetic Scheme for (A) PEG- $b$-p(DMAEMA-co-AMA) copolymer within in polymer series 1 and (B) p(DMAEMA-co-AMA) and p(DEAEMA-co-AMA) copolymers within polymer series 2. 


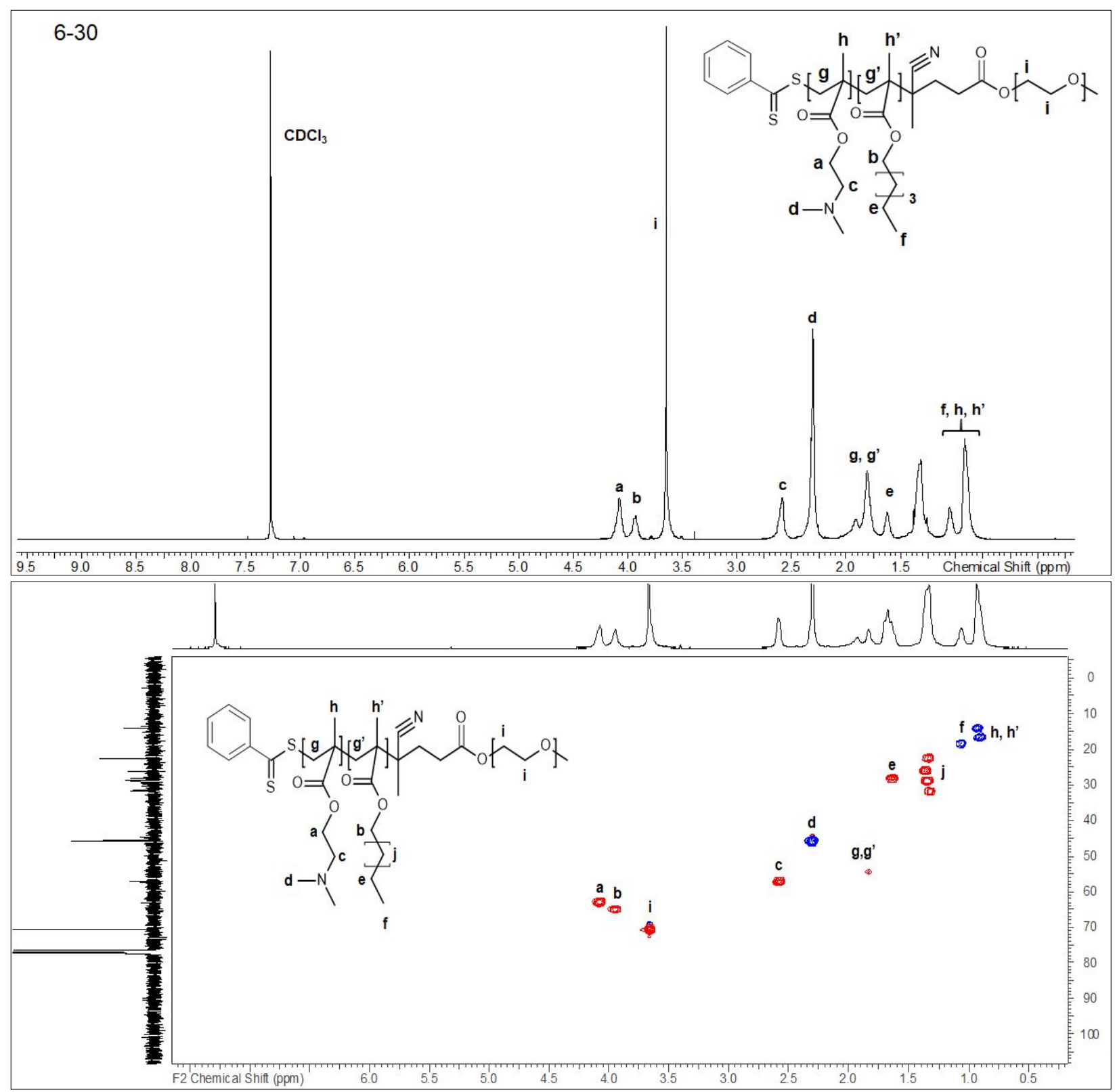

Figure S1. ${ }^{1} \mathrm{H}-\mathrm{NMR}$ (top) and HSQC (bottom) spectra in $\mathrm{CDCl}_{3}$ of "6-30" PEG- $b$-p(DMAEMA-co-AMA) copolymer from polymer series 1 . 


\begin{tabular}{|c|c|c|c|c|c|c|c|}
\hline Polymer Name & $\begin{array}{c}\text { Side } \\
\text { chain } \\
\text { length } \\
\text { [n] }\end{array}$ & $\begin{array}{c}\text { AMA } \\
\text { content } \\
{[\%]}\end{array}$ & Total $M_{n}$ & $\begin{array}{c}\mathrm{M}_{\mathrm{n}} \text { 2nd } \\
\text { block }\end{array}$ & $\begin{array}{c}\text { DP } \\
\text { DMAEMA }\end{array}$ & $\begin{array}{c}\text { DP } \\
\mathbf{A}_{\mathbf{n}} \mathbf{M A}\end{array}$ & $\begin{array}{c}\text { Fraction } \\
\mathbf{A}_{\mathbf{n}} \mathbf{M A}\end{array}$ \\
\hline $2-30$ & 2 & 30 & 32274 & 22274 & 112.4 & 45.3 & 0.287 \\
\hline $2-60$ & 2 & 60 & 31486 & 21486 & 71.4 & 93.1 & 0.566 \\
\hline 4-30 & 4 & 30 & 32383 & 22383 & 105.6 & 44.4 & 0.296 \\
\hline $4-40$ & 4 & 40 & 34203 & 24203 & 97.7 & 65.6 & 0.402 \\
\hline $4-50$ & 4 & 50 & 39050 & 29050 & 95.3 & 98.9 & 0.509 \\
\hline $4-60$ & 4 & 60 & 34856 & 24856 & 71.2 & 98.6 & 0.581 \\
\hline $6-30$ & 6 & 30 & 37061 & 27061 & 113.0 & 58.0 & 0.339 \\
\hline $6-40$ & 6 & 40 & 36127 & 26127 & 92.2 & 71.1 & 0.436 \\
\hline $6-50$ & 6 & 50 & 33966 & 23966 & 71.9 & 76.6 & 0.516 \\
\hline $8-30$ & 8 & 30 & 35295 & 25295 & 102.9 & 48.6 & 0.321 \\
\hline $8-40$ & 8 & 40 & 38701 & 28701 & 97.9 & 69.6 & 0.416 \\
\hline $8-50$ & 8 & 50 & 38409 & 28409 & 78.2 & 83.3 & 0.516 \\
\hline $10-30$ & 10 & 30 & 39043 & 29043 & 106.8 & 56.4 & 0.346 \\
\hline $10-40$ & 10 & 40 & 40679 & 30679 & 106.5 & 63.8 & 0.375 \\
\hline $10-50$ & 10 & 50 & 46868 & 36868 & 99.2 & 96.0 & 0.492 \\
\hline $12-30$ & 12 & 30 & 48079 & 38079 & 143.5 & 63.8 & 0.308 \\
\hline $12-40$ & 12 & 40 & 37292 & 27292 & 84.7 & 56.6 & 0.401 \\
\hline $12-50$ & 12 & 50 & 41495 & 31495 & 77.7 & 77.4 & 0.499 \\
\hline $12-60$ & 12 & 60 & 39954 & 29954 & 53.9 & 85.5 & 0.613 \\
\hline
\end{tabular}

Table S1. Characteristics in polymer series 1 (composition library) ranging from $30 \%$ to $60 \%$ (\%) alkyl methacrylate (AMA) that contain various lengths of alkyl side chains (n). DP = degree of polymerization (total monomeric units). 


\begin{tabular}{cccccccc}
\hline Polymer & $\begin{array}{c}\text { PEG } \\
\text { MW }\end{array}$ & $\begin{array}{c}\text { Total } \\
\text { Mn }\end{array}$ & Total DP & $\begin{array}{c}\text { Fraction } \\
\text { DMAEMA }\end{array}$ & $\begin{array}{c}\text { Fraction } \\
\text { DEAEMA }\end{array}$ & $\begin{array}{c}\text { Fraction } \\
\text { BMA }\end{array}$ & PDI \\
\hline DB6375 & - & 6375 & 38 & 0.6 & 0 & 0.4 & 1.081 \\
DB7074 & - & 7074 & 67.45 & 0.6 & 0 & 0.4 & 1.326 \\
DB17910 & - & 17910 & 119 & 0.6 & 0 & 0.4 & 1.009 \\
DB25170 & - & 25170 & 162.98 & 0.6 & 0 & 0.4 & 1.032 \\
EB10250 & - & 10250 & 35.6 & 0 & 0.6 & 0.4 & 1.091 \\
EB10970 & - & 10970 & 59.5 & 0 & 0.6 & 0.4 & 1.012 \\
EB17610 & - & 17610 & 92.75 & 0 & 0.6 & 0.4 & 1.038 \\
EB23060 & - & 23060 & 127.4 & 0 & 0.6 & 0.4 & 1.017 \\
PEG-DB9542 & 2000 & 9542 & 33.25 & 0.6 & 0 & 0.4 & 1.027 \\
PEG-DB12490 & 2000 & 12490 & 63.65 & 0.6 & 0 & 0.4 & 1.017 \\
PEG-DB22220 & 2000 & 22220 & 88.2 & 0.6 & 0 & 0.4 & 1.031 \\
PEG-DB21990 & 2000 & 21990 & 120.83 & 0.6 & 0 & 0.4 & 1.01 \\
PEG-EB9187 & 2000 & 9187 & 28.5 & 0 & 0.6 & 0.4 & 1.006 \\
PEG-EB12350 & 2000 & 12350 & 47.6 & 0 & 0.6 & 0.4 & 1.016 \\
PEG-EB20900 & 2000 & 20900 & 99.75 & 0 & 0.6 & 0.4 & 1.019 \\
PEG-EB18280 & 2000 & 18280 & 109.2 & 0 & 0.6 & 0.4 & 1.04
\end{tabular}

Table S2. Characteristics in the polymer series 2 (MW library) with an approximately 60:40 ratio of DMAEMA (D) or DEAEMA (E) to BMA (B) monomers in the absence and presence of an initial PEG block $(2,000 \mathrm{~g} / \mathrm{mol})$. Mn $=$ molecular weight and $\mathrm{DP}=$ degree of polymerization (total monomeric units). 

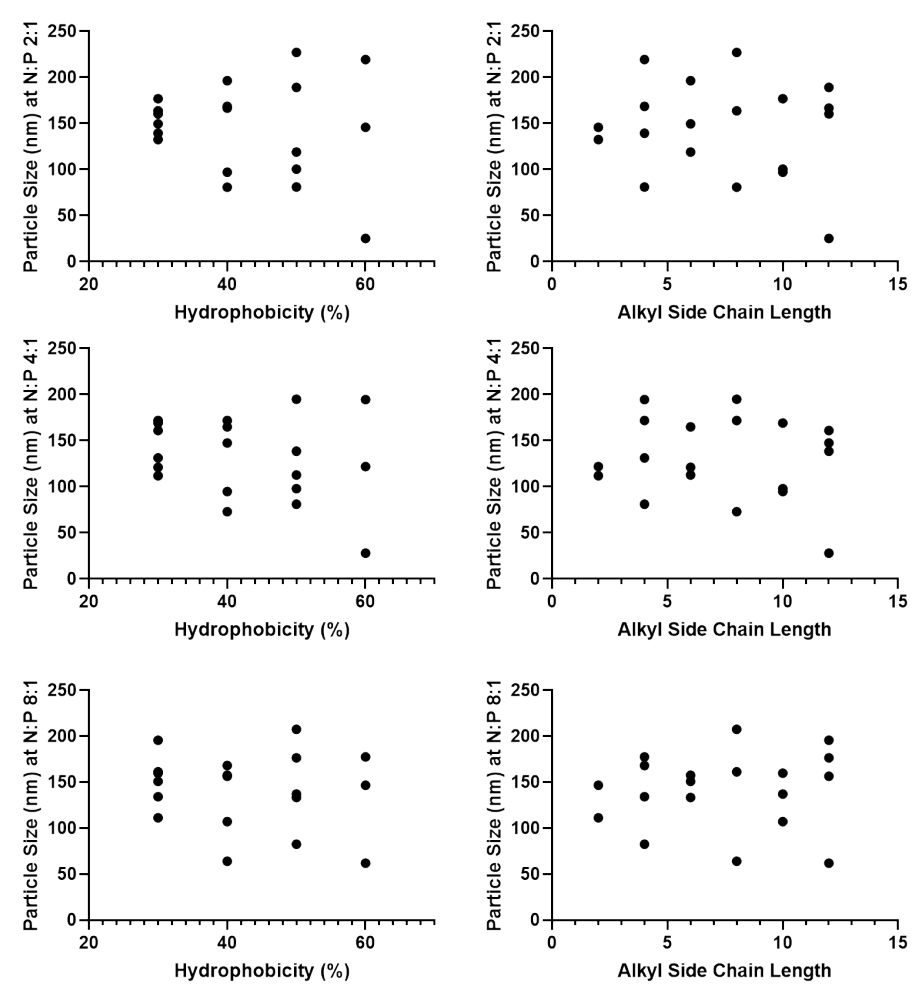

\begin{tabular}{|c|c|c|c|c|}
\hline & N:P & Pearson Value & P Value & $\begin{array}{c}\text { Significant (alpha = } \\
\mathbf{0 . 0 5} \text { ? }\end{array}$ \\
\hline \multirow{3}{*}{ Hydrophobicity vs. Particle Size } & $2: 1$ & -0.14 & 0.28 & No \\
\cline { 2 - 4 } & $4: 1$ & -0.24 & 0.16 & No \\
\cline { 2 - 5 } & $8: 1$ & -0.14 & 0.29 & No \\
\hline \multirow{2}{*}{ Alkyl Side Chain Length vs. Particle } & $2: 1$ & -0.12 & 0.31 & No \\
\cline { 2 - 5 } Size & $4: 1$ & -0.12 & 0.32 & No \\
\cline { 2 - 5 } & $8: 1$ & 0.07 & 0.38 & No \\
\hline
\end{tabular}

Figure S2. Pearson correlation analysis of hydrophobicity and alkyl chain length vs. particle size within polymer series 1 at 2:1, 4:1, and 8:1 N:P ratios. 


\begin{tabular}{|c|c|c|c|c|}
\hline \multirow{2}{*}{ Polymer } & \multicolumn{2}{|c|}{ Dz (nm) } & \multicolumn{2}{c}{ PDI } \\
\hline & Formulation Method & \multicolumn{2}{|c}{ Formulation Method } \\
\hline $\mathbf{4 - 4 0}$ & Vortex & HT & Vortex & HT \\
\hline $\mathbf{4 - 5 0}$ & 87.7 & 161.3 & 0.167 & 0.218 \\
\hline $\mathbf{4 - 6 0}$ & 98.6 & 159.9 & 0.202 & 0.2305 \\
\hline $\mathbf{6 - 3 0}$ & 122.1 & 195.8 & 0.229 & 0.2245 \\
\hline $\mathbf{6 - 4 0}$ & 61.9 & 168.3 & 0.125 & 0.222 \\
\hline $\mathbf{6 - 5 0}$ & 69.6 & 157.6 & 0.228 & 0.268 \\
\hline $\mathbf{8 - 3 0}$ & 68.1 & 64.1 & 0.219 & 0.2105 \\
\hline $\mathbf{8 - 4 0}$ & 48.0 & 107.1 & 0.187 & 0.23 \\
\hline $\mathbf{8 - 5 0}$ & 58.7 & 156.6 & 0.145 & 0.293 \\
\hline $\mathbf{1 0 - 3 0}$ & 57.7 & 82.6 & 0.172 & 0.2125 \\
\hline $\mathbf{4}$ & 50.5 & 133.4 & 0.106 & 0.241 \\
\hline
\end{tabular}
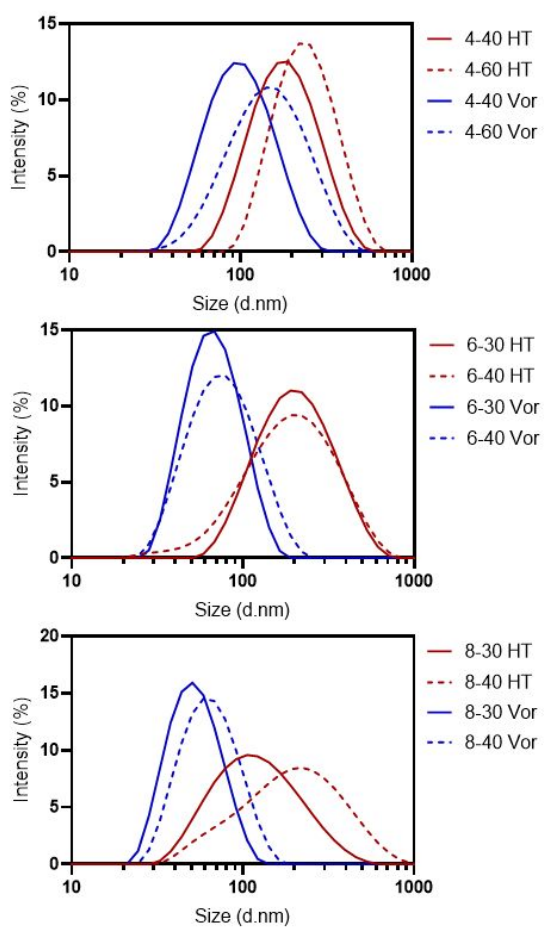

Table S3. Hydrodynamic diameter of polyplexes prepared by conventional preparation (vortexed for $10 \mathrm{sec}$, incubated for at least $15 \mathrm{~min}$ ) (Vortex [blue]) and robot pipetting (HT [red]) at 8:1 N:P ratio. DLS were measured with Zetasizer Nano ZS (Malvern Instruments, Herrenberg, Germany) via cuvettes and plates at final mRNA concentration of 25 $\mu \mathrm{g} / \mathrm{mL}$. 

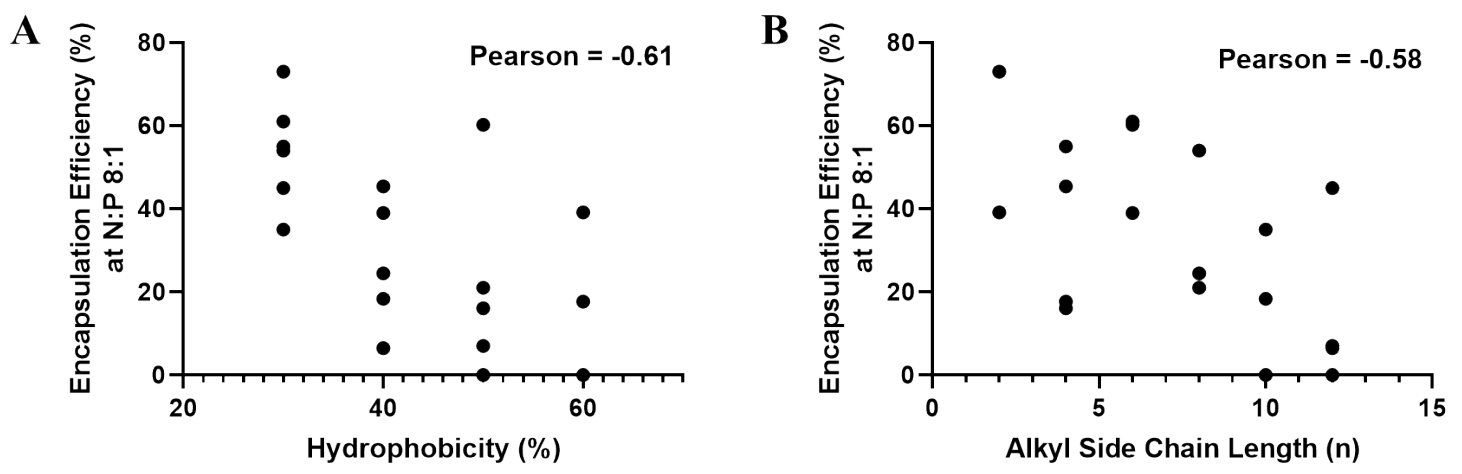

Figure S3. Pearson correlation between encapsulation efficiency of mRNA polymer carriers versus (A) hydrophobic percentage and (B) alkyl chain length within each copolymer used. $\mathrm{p}<0.005$. 


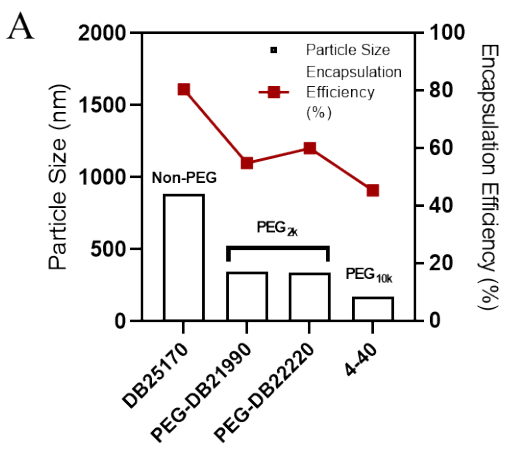

Polymer Vector

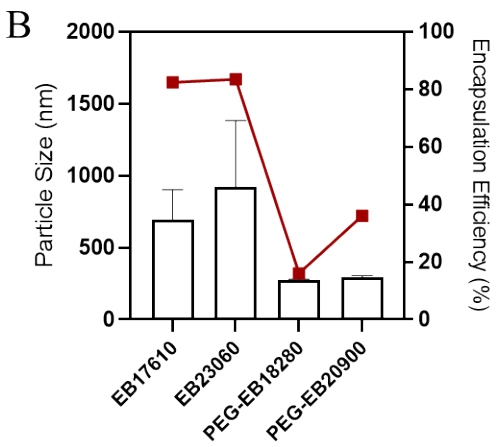

Polymer Vector

$\mathrm{C}$

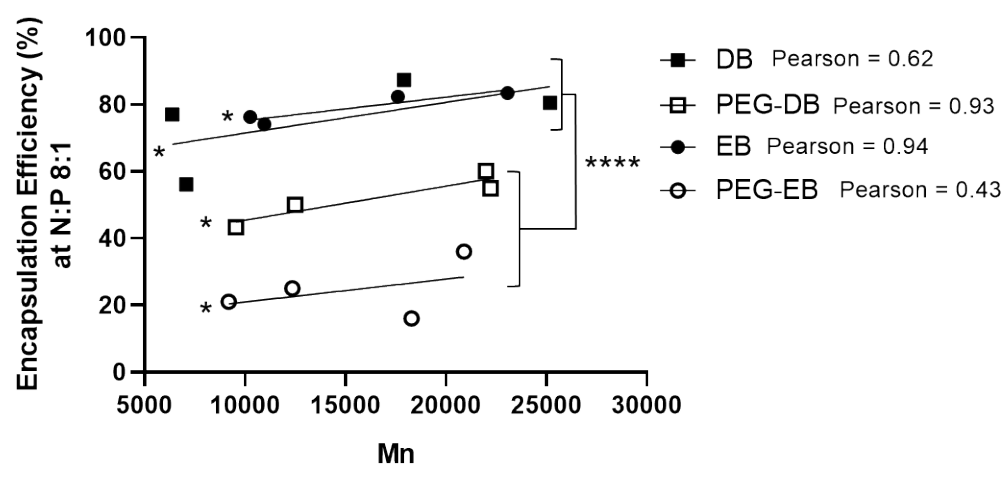

Figure S4. SAR trends of polyplex size and cargo encapsulation depicting influence of PEG addition to (A) DMAEMA (DB) and (B) DEAEMA (EB) copolymers with comparable amphiphilic block segments at 8:1 N:P ratio. (C) Pearson correlation between encapsulation efficiency of mRNA polymer carriers in series 2 versus molecular weight. $*=\mathrm{p}<0.05$ and $* * * *=\mathrm{p}<0.0001$. 
A
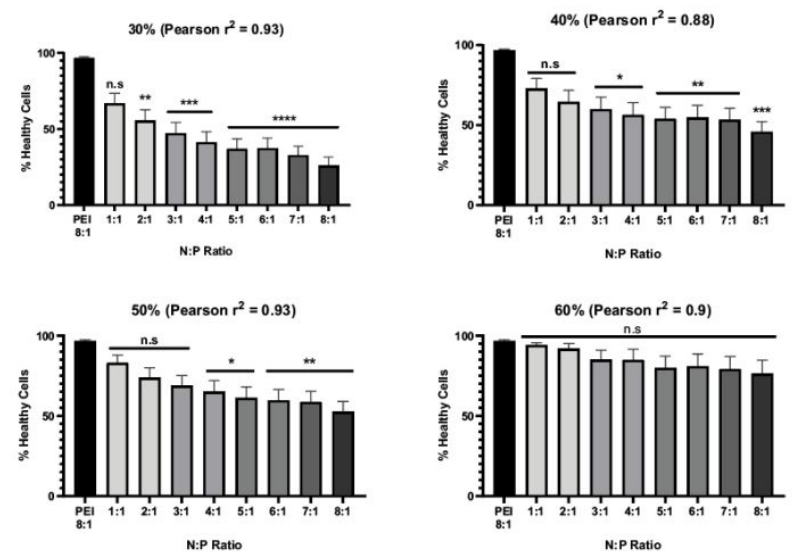

B
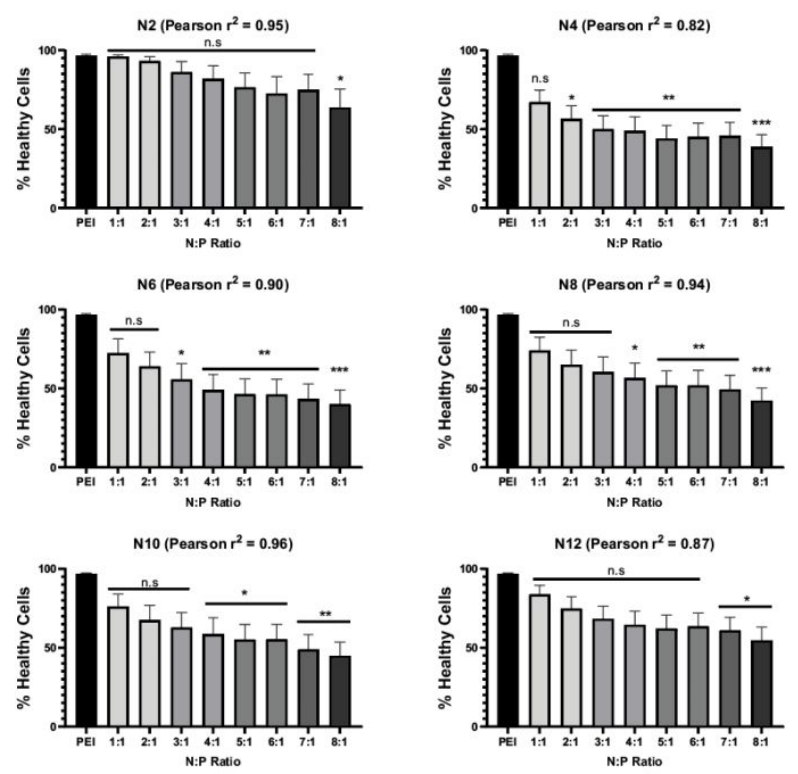

C
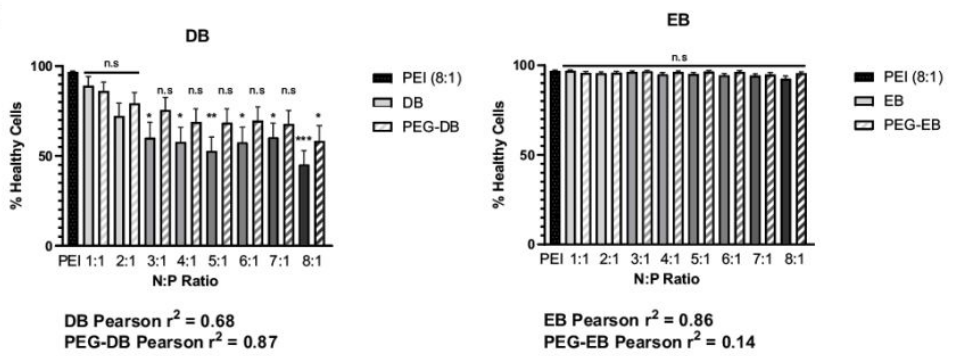

Figure S5. Cytotoxicity of polyplexes with varying amphilicity, alkyl side chain length, and cation type. By increasing N:P ratios, cell health in Huh7 cells was shown to be directly affected by (A) the concentration of hydrophobic monomers and (B) alkyl side chain lengths. (C) Cytotoxicity trends of copolymers $(\mathrm{MW}=\sim 9000$ to 22500) containing cationic DMAEMA (DB) and DEAEMA (EB) monomers in the presence and absence of an initial PEG block. Each formulation $(1 \mu \mathrm{g} / \mathrm{mL})$ is loaded with mRNA (4:1 GFP:Cy5) was dosed to Huh7 cells for $24 \mathrm{~h}$. Cytotoxicity is represented as the percent healthy cells from all nuclei detected, and graphs show mean values \pm SEM from $n=3$ independent experiments. Significance was determined in A-C by one-way ANOVA followed by Dunnett's multiple comparisons test to the PEI control where $*=\mathrm{p}<0.05, * *=\mathrm{p}<0.01, * * *=\mathrm{p}<0.001, * * * *=\mathrm{p}<0.0001$, n.s $=$ not significant. 

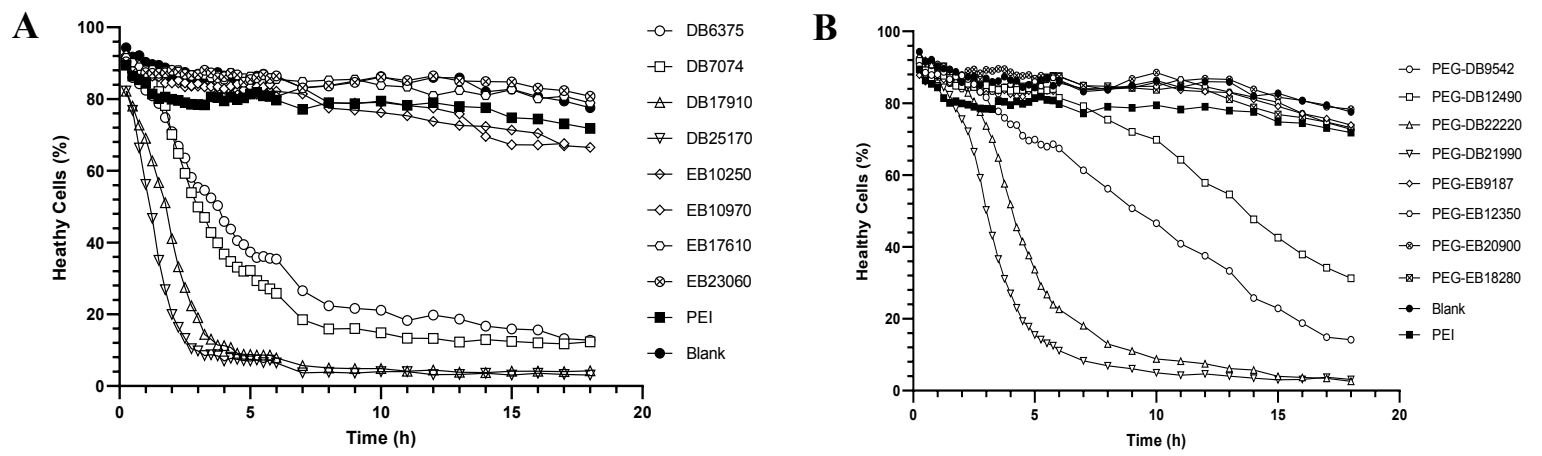

Figure S6. Live-cell cytotoxicity profiles of (A) p(DMAEMA/DEAEMA-co-BMA) and (B) $\mathrm{PEG}_{2 \mathrm{k}}-b$ $\mathrm{p}$ (DMAEMA/DEAEMA-co-BMA) polyplexes. Cell health was determined through confocal imaging of the nuclei morphology and size. 


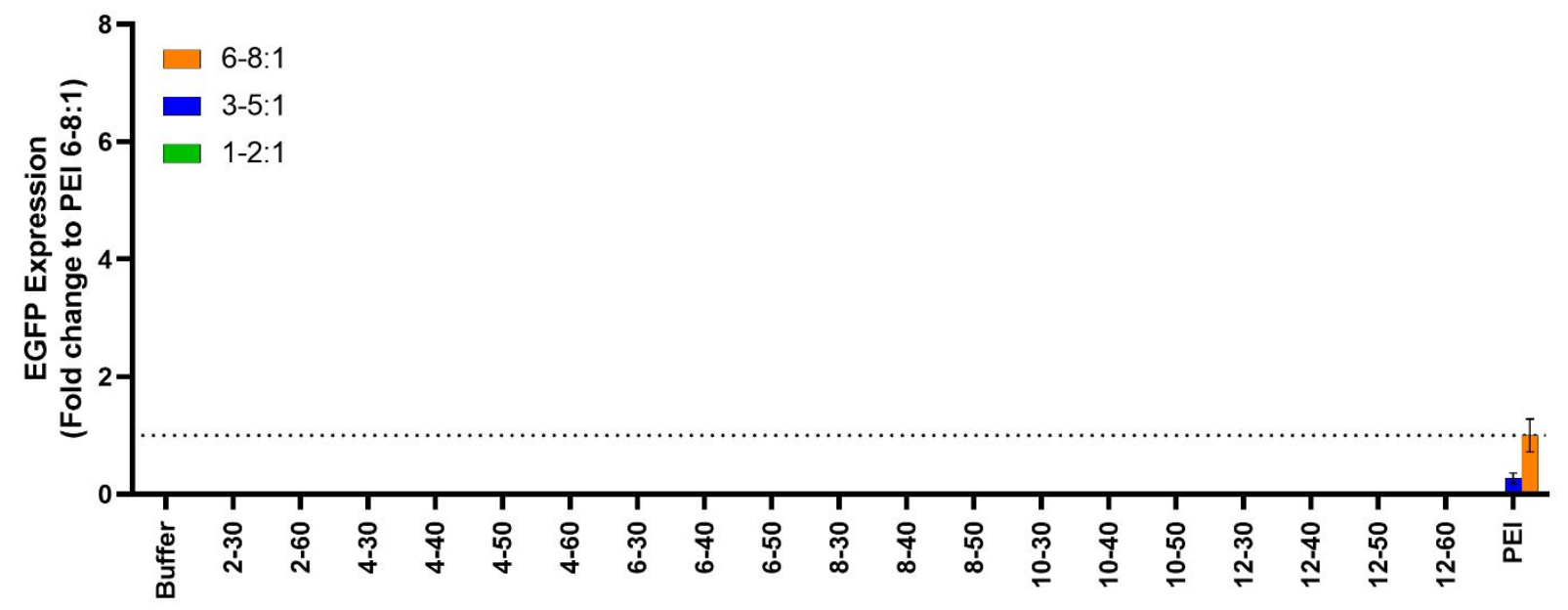

Figure S7. mRNA transfection efficiencies in Huh7 cells at different ranges of N:P ratios of PEG10k-b-p(DMAEMAco-AMA). Quantitation of polyplex efficiency of polymer set 1 in Huh7 cells following $24 \mathrm{~h} \mathrm{dosing} \mathrm{with} 1 \mu \mathrm{g} / \mathrm{mL}$ EGFP mRNA via indicated polyplexes and binned for indicated N:P ratios (determined by percentage of cells positively transfected multiplied by mean cellular EGFP intensity). Bars represent mean values normalized to the PEI control (6-8:1 N:P) from a minimum of $n=3$ independent experiments \pm SEM. 


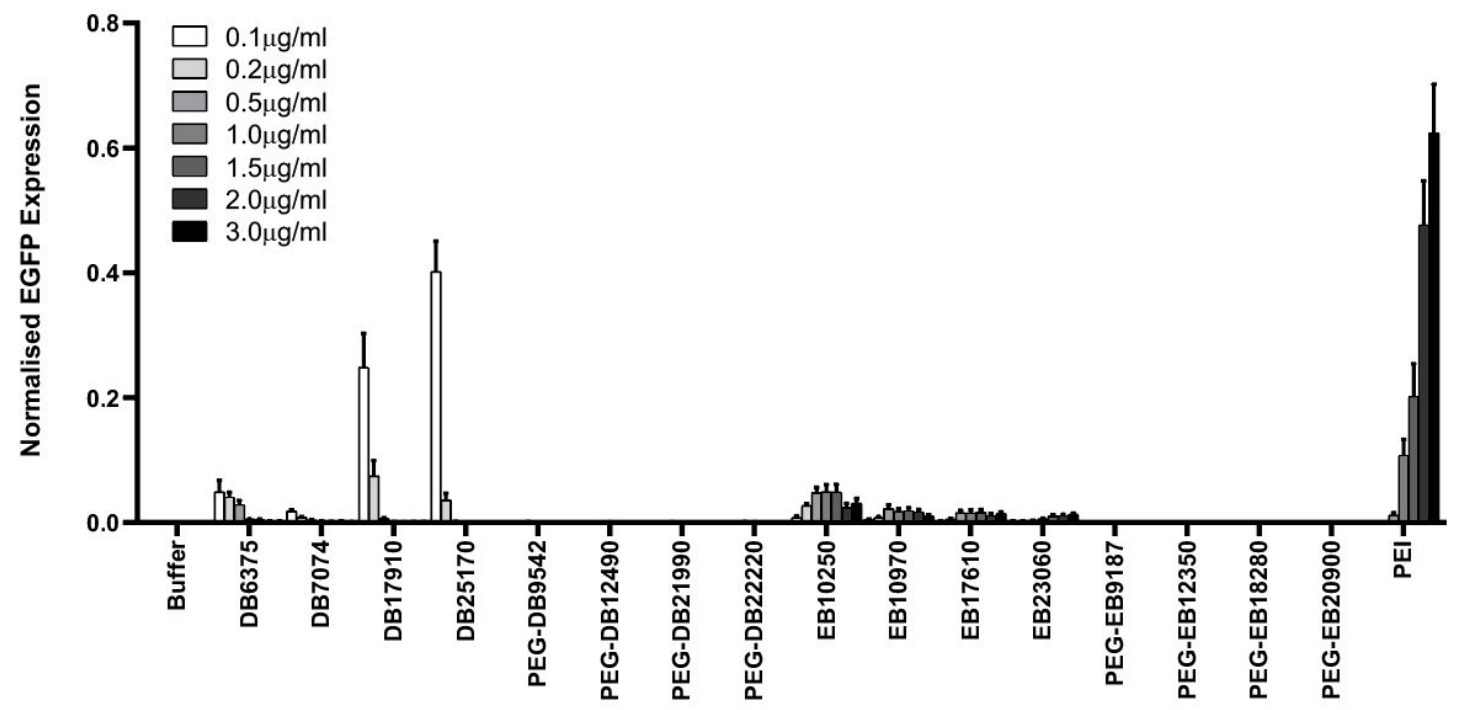

Polymer Name

b

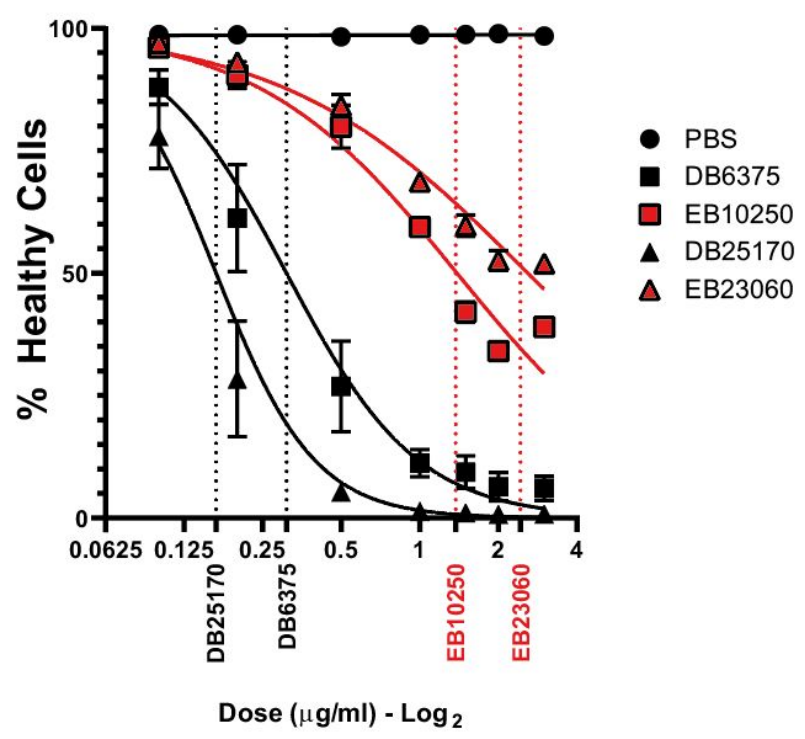

C

\begin{tabular}{|l|c|}
\hline \multicolumn{1}{|c|}{ Polymer } & LC50 $(\boldsymbol{\mu g} / \mathbf{m l})$ \\
\hline DB6375 & 0.3087 \\
\hline DB7074 & 0.2648 \\
\hline DB17910 & 0.1391 \\
\hline DB25170 & 0.1659 \\
\hline PEG-DB9542 & 0.4959 \\
\hline PEG-DB12490 & 1.115 \\
\hline PEG-DB21990 & 0.2105 \\
\hline PEG-DB22220 & 0.2484 \\
\hline EB10250 & 1.374 \\
\hline EB10970 & 1.788 \\
\hline EB17610 & 2.27 \\
\hline EB23060 & 2.43 \\
\hline PEG-EB9187 & $>3$ \\
\hline PEG-EB12350 & $>3$ \\
\hline PEG-EB18280 & $>3$ \\
\hline PEG-EB20900 & $>3$ \\
\hline PEI & $>3$ \\
\hline Buffer & $>3$ \\
\hline
\end{tabular}

Figure S8. Dose dependent studies of the MW polymer library consisting of cationic DMAEMA (DB) and DEAEMA (EB) monomers. EGFP transfection and cell death of indicated polyplexes were examined through microscopy by dosing Huh7 cells at a dose range of $0.1-3 \mu \mathrm{g} / \mathrm{mL}$ (all formulated at 8:1 N:P). (A) EGFP expression and (B) cytotoxicity $\left(\mathrm{LC}_{50}\right)$ of all polyplexes was examined $24 \mathrm{hrs}$ after initial dosing. (C) All polyplexes exhibit $\mathrm{LC}_{50}$ values such that percentage of unhealthy/dead cells were determined through microscopy and concentrations were found after line fitting. Bars represent mean values from $n=3$ independent experiments \pm SEM. Significance was determined by twoway ANOVA followed by Dunnett's multiple comparison test to the Buffer control. Significance is denoted by $*=p$ $<0.05, * *=\mathrm{p}<0.01, * * *=\mathrm{p}<0.001, * * * *=\mathrm{p}<0.0001, \mathrm{n} . \mathrm{s}=$ not significant. 